\title{
Characteristics of proposed 3 and 4 telescope configurations for Darwin and TPF-I
}

\author{
Kaltenegger L., ${ }^{1,2} \dagger$ and Fridlund M. ${ }^{2}$ \\ ${ }^{1}$ Harvard-Smithsonian Center for Astrophysics, 60 Garden St, Cambridge, MA 02138, USA \\ email: lkaltenegger@cfa.harvard.edu \\ ${ }^{2}$ European Space Agency ESTEC, P.O.Box 299, NL-2200AG Noordwijk, The Netherlands \\ email:Malcolm.Fridlund@esa.int
}

\begin{abstract}
The Darwin and TPF-I missions are Infrared free flying interferometer missions based on nulling interferometry. Their main objective is to detect and characterize other Earth-like planets, analyze the composition of their atmospheres and their capability to sustain life, as we know it. Darwin and TPF-I are currently in study phase. A number of mission architectures of 3 and 4 free flying telescopes are evaluated on the basis of the interferometer's response, ability to distinguish multiple planet signatures and starlight rejection capabilities. The characteristics of the new configurations are compared also to the former, more complex Bowtie baseline architectures as well as evaluated on base of their science capability.
\end{abstract}

Keywords. astrobiology, extrasolar planets, Earth, interferometers, nulling.

\section{Introduction}

The closing years of the 20th century have allowed us, for the first time, to seriously discuss interferometric instruments deployed in space achieving unprecedented spatial resolution. And thus the direct detection of Earth-like exoplanets orbiting nearby stars, and the search for key tracers of life in their atmospheres, are high-priority objectives in the long-term science plan of ESA as well as NASA. Both Agencies are working on the definition of the instruments that will meet this challenge, Darwin and Terrestrial Planet Finder (TPF) respectively with a foreseen launch in 2015+. The baseline mission duration is 5 years, extendable to 10 years in an L2 orbit. Operating in the infrared band requires that all optical components are cooled to roughly $40 \mathrm{~K}$, this is achieved by passive cooling. Only the detector requires active cooling. Darwin is a major element in the Cosmic Vision 2020 program of the European Space Agency. It has the explicit purpose of detecting other Earth-like worlds, analyze their characteristics, determine the composition of their atmospheres and investigate their capability to sustain life as we know it. The Darwin mission is envisioned as four free flying spacecraft including one beam-combining spacecraft. The beam combiner and the telescope spacecraft fly in one plane with each telescope spacecraft at the same distance from the beam combiner. The resolution of the interferometer is adjusted by changing the distance between the telescope spacecrafts. A similar activity has been taking place in the United States within the context of NASA's Origins program. A science collaboration has already been established. The missions are currently in definition phase, here some characteristics of the designs are reviewed. We present the different new configuration architectures under investigation.

$\dagger$ Present address:Harvard-Smithsonian Center for Astrophysics, MS20, 60 Garden Street, Cambridge, MA 02138, USA 


\section{Nulling Interferometry}

The main problem in the direct detection of an exo-planet of a size comparable to our own Earth and located at a similar distance from its own star is contrast and dynamic range. A central star like our Sun $(\mathrm{G} 2 \mathrm{~V})$ outshines an Earth-like planet in the visual wavelength range by a factor of at least $10^{9}$. Going to the mid-infrared alleviates this problem, because the planet's thermal emission peaks at $10 \mu \mathrm{m}$. Even at these wavelengths, the contrast is more than a factor of $10^{6}$. Analysis of the planetary light requires that the stellar light is suppressed to a high degree. In the IR this is done by a technique called nulling interferometry, in essence this means that achromatic phase shifts are applied to the beams collected by individual telescopes before recombination such that the on-axis light, i.e. stellar light, is cancelled by destructive interference, while the much weaker planetary light emitted at a certain off-axis angle interferes constructively. By keeping a star in the center of the image plane, coronography is realized without the presence of a physical mask. The output of the system can be described by an angular transmission map (TM) featuring interference fringes, with a sharp null (destructive interfered area) in the center of the map. The TM gives the interferometer's intensity response as a function of the sky coordinates. The modulation map (MM) is the difference between the recorded outputs on detector A and detector B. The stellar signal is nulled out only on the optical axis. A leakage of photons out of the central null exists because the star has a finite photospheric disk. That leakage is a very important noise source. The actual shape and transmission properties of the pattern are a function of the number of telescopes, configuration, and the distance between the telescopes (see e.g, Absil (2001),Kaltenegger (2004)). Information about the distribution of planets in the target star system can be recovered by modulation of that signal. Figure 1 shows the TM, MM and signal modulation for a simulated Solar System (Earth, Venus, Mars, Jupiter shown) for different configurations. No spatial information is extracted in a single exposure. Rotation modulates the interferometer output intensity as a planet passes in and out of the dark fringes. From the intensity and actual pattern of this modulation one can derive the planet's parameters.

\subsection{Architecture design}

The original Darwin mission concept was optimized for high stellar rejection to focus on observing the closest stars for planetary companions. This led to a baseline concept of a free flying configuration with 6 collector telescopes (Bowtie configuration) of $1.5 \mathrm{~m}$ diameter and a central beam combiner (see e.g. Absil (2001) for an overview). Optimizing the mission for nearby stars is not the same as optimizing the mission performance for its overall star sample of a minimum of 165 stars out of the DARWIN target catalogue Kaltenegger, Eiroa, Stankov et al. (2005). Recent analyses show that the starlight rejection criteria can be relaxed while still maintaining a target sample of 165 stars. The integration time for nearby stars increases, but the overall mission performance does not degrade significantly. A number of these alternative mission architectures have been evaluated on the basis of interferometer response, achievable modulation efficiency, number of telescopes and starlight rejection capabilities (see Kaltenegger \& Karlsson (2004) and Lay \& Dubovitsky (2004)). Accordingly, candidate Darwin and TPFI configurations use three (Three telescope Nuller (TTN) see Karlsson, Wallner \& Perdigues Armengol (2003)) or four (X-array, see Lay \& Dubovitsky (2004) telescopes of $3.5 \mathrm{~m}$ diameter, reducing complexity and cost of the mission. Three telescopes is the minimum number of telescopes needed for an interferometer mission that uses rapid signal modulation to detect a planet in the high background noise. An array with 6 telescopes like the Bowtie 
achieves better performance in starlight rejection than an array with 3 or 4 telescopes, but adds complexity and cost to the mission. Furthermore the maximum modulation efficiency is $100 \%, 93 \%$ while only $70 \%$ for the X-array, TTN and Bowtie, repectively. The mean modulation efficiency is $26 \%, 32 \%$ and only $16 \%$ for the X-array, TTN and Bowtie, repectively. These numbers show a performance increase for the 3 and 4 telescope architectures in comparison to the Bowtie. Modulation efficiency is a measure of the efficiency with which planet photons are converted into output signal. The maximum modulation efficiency sets the performance for the mission's spectroscopy phase, the mean modulation the efficiency for the search phase. The higher the modulation efficiency, the better the overall performance of the architecture.

The output beam comprises all information from a specific set of sources on the sky, as well as the background. In an ideal scenario with only a single planet around its host star and no other disturbing sources, such as extrasolar zodiacal dust in the target system, the detection of a positive flux would imply that a planet is present, if the star is well and truly 'nulled out'. In real observations, several factors affect the signal to noise in a detrimental way. This has been analyzed by different groups e.g. Lay \& Dubovitsky (2004) and Kaltenegger \& Karlsson (2004). Figure 1 shows that it is essential to apply spectral channels to distinguish the signal from multiple planets due to their distinct signature as a function of distance from their star, wavelength and rotation of the array (shown here, Venus, Earth, Mars and Jupiter). The simulations of our own Solar system in Figure 1 show that Jupiter's signal is seen at higher frequency over a full rotation of the array than an Earth-like planet. Thus a Jupiter will be easily distinguished. Our simulations show that Venus and Earth are the two planets in our own solar system that are most likely to be confused. Here the different architectures show their capability to disentangle multiple planetary signals. As seen in Figure 1 the rectangular TTN and the X-array perform the best in this respect. Note that these calculations do not take noise into account. By increasing the distance between the telescopes any modulation pattern can be made finer, but that generally increases the stellar leakage. The $\mathrm{X}$-array concept has an independent nulling and imaging baseline (the imaging baseline controls the resolution of the modulation map). It is the distance between the two sub-nulling Interferometers in the X-array concept. Preliminary investigations show that the 4 telescope array (Xarray) has a better performance with respect to distinguishing multiple planets due to a finer grid in its modulation pattern Lay \& Dubovitsky (2004). The TTN design is a concept that combines the nulling and imaging baseline. Increasing the imaging baseline to gain resolution thus also increases the nulling baseline what decreases the extent of the central null as the whole modulation map scales and thus increases the stellar leakage. Recent simulations by Velusamy \& Marsh (2005) and Thiebaut \& Mugnier (2005) argue that high spatial resolution is not needed to disentangle multiple planets, avoiding the difference in performance due to leakage increase for the TTN at larger baselines. These results would indicate similar performance of the three and four telescope architectures.

\section{Conclusions}

A number of mission architectures of 3 and 4 free-flying telescopes are evaluated on the basis of the interferometer's response, modulation efficiency, ability to distinguish multiple planet signatures and starlight rejection capabilities. Figure 1 shows the characteristics of the alternative mission architectures. Even though the starlight rejection properties of the Bowtie configuration is outstanding in the comparison, the higher mean modulation efficiency of the TTN configuration and the X-array counteracts that superiority. An additional factor in mission design and complexity is the number of telescopes 


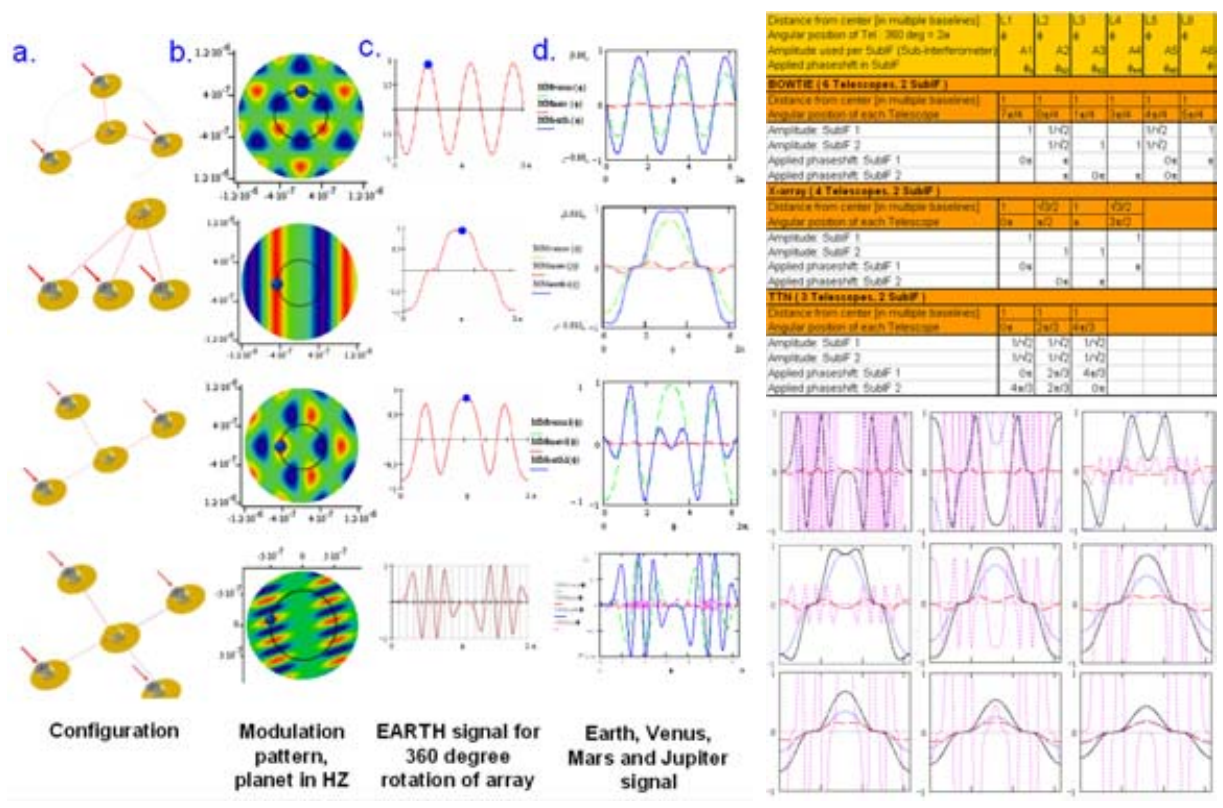

Figure 1. a) Telescope Array Configuration b) Modulation pattern generated by the array c) modulation of the signal of an Earth-like planet at 1AU over a full 360 degree rotation of the array. d) Modulation of the signal from multiple planets (simulation of our Solar System, planets shown: Venus, Earth, Mars and Jupiter). Different frequency of the signal from different planets allows to disentangle the signal from multiple planets. Configurations shown: upper panel to lower panel: 1) equilateral triangle, 2) linear, and 3) rectangular triangle Three Telescope Nuller (TTN) and 4) X array. Right upper panel: Table with architecture characteristics. Right lower panel: The signal of multiple planets in our solar system (Venus, Earth, Mars and Jupiter) recorded in different spectral channels over a whole rotation of the array for the linear TTN configuration (assuming no noise). The high frequency signal shown is Jupiter's. The panels show the monochromatic signal per channel from $4 \mu \mathrm{m}$ (left) to $20 \mu \mathrm{m}$ (right) in steps of $2 \mu \mathrm{m}$.

used. Here the Bowtie has a big disadvantage, as it needs 6 telescopes. Figure 1 shows that it is essential to apply spectral channels to distinguish the signal from multiple planets due to their distinct signature as a function of distance from their star, wavelength and rotation of the array (shown here, Venus, Earth, Mars and Jupiter). Here the different architectures show their capability to disentangle multiple planetary signals. As seen in Figure 1 the rectangular TTN and the X-array perform the best in this respect. Recent simulations by Velusamy et al. and Thiebaut et al. (these proceedings) argue that high spatial resolution is not needed to disentangle multiple planets which would lead to similar performance of the three and four telescope architectures.

\section{References}

Absil O. 2001, PhD thesis, University of Liege, Belgium

Dubovitsky, S. \& Lay, O.P. 2004, SPIE 5491, pp. 284

Lay, O.P. \& Dubovitsky, S. 2004, SPIE 5491, pp. 874

Kaltenegger, L., Eiroa, C., Stankov, A., \& Fridlund, M. 2005, these proceedings

Kaltenegger, L. \& Karlsson, A. 2004, SPIE 5491, pp. 275

Kaltenegger, L. 2004, PhD thesis, Karl-Franzens University Graz, Austria

Karlsson, A., Wallner, O. \& Perdigues Armengol, J. M. 2004, SPIE 5491, pp. 831

Velusamy, T. \& Marsh, K. 2005, these proceedings

Thiebaut, E. \& Mugnier, L. 2005, these proceedings 